\title{
Surgical treatment of hallux rigidus. Review of foreign publications
}

\author{
D.V. Ilchenko, A.A. Kardanov, A.V. Korolev
}

European Clinic of Sports Traumatology and Orthopaedics, Moscow, Russian Federation

The article presents an overview of foreign publications on surgical treatment of hallux rigidus with comparison among different available surgical approaches, good evidence to support most effective modalities and perspective surgical treatment options for the condition.

Keywords: hallux rigidus, first metatarsophalangeal joint arthritis, cheilectomy, arthrodesis, arthroplasty, osteotomy

Hallux rigidus is the most common degenerative arthritic condition in the foot affecting $2.5-7.8 \%$ of people over the age of $50[1,2,3]$. The pathological condition was first described by Nicoladini in 1881, and as the hallux flexus in 1887 by Davies-Colley. In his description of the condition, he discussed a plantarflexed posture of the first phalanx $(\mathrm{P} 1)$ relative to the first metatarsal head (M1). The actual term "hallux rigidus" (HR) was coined by Cotterill [4] in 1888 describing pain at dorsiflexion in the first metatarsophalageal joint (MTPJ) and has remained the most common term used today in the medical literature. To this time the true potential etiologies for the development of hallux rigidus remain in question. The predisposing factors may include an excessively long M1, the elevated posture of the first metatarsal head (metatarsus primus elevatus), osteochondritis dissecans of the first MTPJ, a hypermobility of the first ray, prolonged immobilization, acute trauma, gout and rheumatoid arthritis [5]. Coughlin and Shurnas examined etological factors of the condition. The authors found that $78 \%$ of unilateral disease was associated with trauma, $95 \%$ of patients with a positive family history had bilateral involvement and $67 \%$ of the patients reported immediate family members affected with HR. No significant correlation was detected between HR and pes planus, pes equinus and hypermobility of the first ray, and metatarsus primus elevatus was shown to improve postoperatively without osteotomy in the shaft. Hallux valgus was identified in conjunction with HR $12 \%$ of the time in the series by Coughlin and Shurnas. An elevated hallux valgus interphalangeus angle was evaluated as an associated clinical and radiological finding of HR seen in $90 \%$ of the time in the series of the authors. They also demonstrated a seemingly high (74\%) incidence of flattened and squared metatarsal heads in a cohort of patients with HR [6, 7].

There have been a great number of treatment modalities offered for HR since the end of the XIXth century. Although the management of HR is initially nonoperative surgical treatment can provide more reliable long-term outcomes for selected group of patients. First surveys of the methods date back to 1887 when Davies and Colley offered resection of the proximal part of P1 and Collier made decompression of the first MTPJ by resecting the head of M1. Then surgical procedures were broadly grouped into two categories: joint sparing (e.g., cheilectomy with or without associated osteotomies) and joint sacrificing/destructing (e.g., arthroplasty or arthrodesis of the first MTPJ). A cheilectomy, as a sustainable option for treating HR was popularized by DuVries in the 1950s [8], and many modifications of cheilectomy have been made.

Although exostectomy of first MTPJ was described earlier, Cochrane suggested the technique as early as 1927 to be combined with plantar capsulotomy and soft tissue release at the base of $\mathrm{P} 1$; later, Nilsonne performed the two procedures that served as a temporary treatment at that time [9]. Since the original description of cheilectomy in literature the procedure has become a popular intervention for the treatment of early stages of HR. The limited series showed that a cheilectomy can provide a very good prognosis (88-95 \%) even in selective advanced cases of HR [4, 10-15]. In 1979, Mann et al. reviewed 20 cheilectomy procedures performed with original DuVries technique. The mean follow-up was

67.8 months, dorsiflexion to the first MTPJ maintained at $30^{\circ}$, and no statistically significant degenerative changes were observed in the operated joint throughout the long term [16]. In 1988, Mann and 
Clanton reported on results of cheilectomy produced for 25 patients (31 feet) with a mean follow-up time period of 56 months. Relief of pain was achieved in 22 patients and 6 patients experienced incidental pain that was rated as a good clinical outcome [16]. Metaanalysis performed by Roukis et al. between 1979 and 2009 included outcomes of 706 cheilectomies with a mean follow-up period of 1.6 years, with $8.8 \%$ undergoing surgical revision. The grade of HR was specified as grade I in $20 \%$ of the cases; grade II, in $14.8 \%$; grade III, in $9.1 \%$ and grade IV, in $55.6 \%$ [17]. In 2003, Coughlin and Shurnas reported on outcomes of 110 patients with HR. A cheilectomy was performed on 80 patients with grades I and II. The mean duration of follow-up was 9.6 years after the cheilectomies. There was significant improvement in dorsiflexion of the first MTPJ, AOFAS scores and postoperative pain following the cheilectomies. $92 \%$ (eighty-six) of the ninety-three cheilectomy procedures were successful in terms of pain relief and function, and 9 patients with grade IV HR required arthrodesis of the first MTPJ at a mean follow-up period of 6.9 years [11].

In 1999, Mulier et al. studied functional results of 22 cheilectomies performed on high-level athletes with Regnauld grade I and grade II HR. The mean follow-up was 5 years. Fourteen patients rated their improvement as excellent, 7 rated it good, and 1 rated it as fair. 75 $\%$ of the athletes could return to their prior or higher level of performance, 5 out of 7 remaining patients gave up for causes unrelated to surgical treatment [18]. Nawoczenski et al. assessed first MTPJ kinematics and loading characteristics following cheilectomy in a prospective study of 15 patients at mid-term follow-up of 1.7 years. First MTP motion was shown to increase but dorsiflexion was still less than normative values. The findings suggested no correlation between restoration of dynamic kinematics and patient's satisfaction with the outcome of surgical treatment and pain relief [19]. Cheilectomy in conjunction with osteotomies of P1 and $\mathrm{M} 1$ can be advocated for decompression to the first MTPJ in HR cases [20]. Closing-wedge osteotomy of the proximal phalanx is known as the Moberg osteotomy aimed to move a limited arc of motion at the affected joint to a more dorsiflexed position decreasing plantar flexion.

The positive effect of the Moberg osteotomy on first MTPJ contact mechanics was experimentally tested by Kim et al. using cadaveric first ray specimens. The Moberg osteotomy was found to lead to a plantar shift in the center of contact pressure on the proximal phalanx surface with no differences in peak pressure and the joint contact area. The Moberg osteotomy was shown to considerably decrease the contact pressure exerting positive effect on clinical manifestations of HR due to cartilage degeneration in first MTPJ being dorsal in most cases [21]. Thomas and Smith reported $99 \%$ positive results with no complication and revision surgeries in 17 patients with grade I and grade II HR treated with the Moberg procedure. The mean followup was 2.5 years [22]. A potential drawback to the use of the Moberg osteotomy was during conversion to MTPJ fusion. The extended position of the proximal phalanx might preclude the use of arthrodesis plates, in terms of an increase in dorsiflexion [23].

A retrospective review of 19 patients with Hattrup and Johnson grades II and III HR managed with a cheilectomy in conjunction with osteotomy of P1 was performed by Lau and Daniels. The mean follow-up period was 2.1 years. Outcome measures demonstrated $87.5 \%$ of patient satisfaction, VAS score of 2.9 points, dorsiflexion significantly improved from $14.1^{\circ}$ to $30.2^{\circ}$ [24]. Outcomes of isolated cheilectomy are comparable with those seen from cheilectomy performed in conjunction with P1 osteotomy. Waizy et al. compared two groups of patients with Regnauld grade I and grade II HR treated with the above procedures.

The mean dorsiflexion in first MTPJ increased in patients with cheilectomy $(\mathrm{n}=23)$ to $24.8^{\circ}$, and excellent results were obtained in $21.7 \%$ of the cases. The mean dorsiflexion increased in patients with cheilectomy and P1 osteotomy $(\mathrm{n}=23)$ to $20.8^{\circ}$, and excellent results were obtained in $32.6 \%$ of the cases. It was concluded that cheilectomy performed standalone or in conjuction with P1 osteotomy is an effective treatment approach for early grades HR [25]. Recommended surgical option for metatarsus primus elevatus being historically considered a primary pathology in association with HR includes a M1 osteotomy to address the deformity and/ or the metatarsal length [7]. Dickerson and colleagues undertook a retrospective analysis of the long-term efficacy of the original Watermann wedge osteotomy of M1 and modified Green-Watermann procedure performed for 32 patients with HR. The average length of follow-up was 4 years. Although $94 \%$ of the patients reported that surgery had significantly relieved their hallux joint pain, $25 \%$ had secondary metatarsalgia [26].The effects of Youngswick osteotomy (much like the Green-Watermann procedure) of the M1 on plantar peak pressure distribution in the forefoot presented for 17 patients (23 feet) were explored by Bryant et al. with the follow-up length of 2 years. Peak pressure was shown to increase beneath the second metatarsal head to $28 \%$ of baseline level [27]. Derner et al. reported the 
findings of a plantar-flexor-shortening M1 osteotomy in treatment of 26 patients with Drago, Oloff and Jacobs grades II and III HR evaluated at a mean follow-up of 2.9 years. At last follow-up, $85 \%$ of patients rated their result as excellent and first MTPJ total range of motion showed the mean increase from $33.3^{\circ}$ to $72.1^{\circ}$. Fifteen percent of patients had postoperative lesser metatarsalgia, and $15 \%$ reported other complications [28]. Malerba and colleagues analyzed 20 patients with grade III HR treated with the Weil M1 osteotomy. The average follow-up period of 11.1 years demonstrated the average AOFAS score increase to 82 , good to excellent results achieved in $95 \%$ of the patients, the mean passive dorsiflexion of first MTPJ improved from $8^{\circ}$ to $44^{\circ}$, with one case of metatarsalgia being the only postoperative complication [29].

Literature review shows a high rate of complications following M1 osteotomy with secondary metatarsalgia being most common. A systematic review performed by Roukis reported a total of $30.5 \%$ of patients developing postoperative metatarsalgia [30]. M1 osteotomy cannot be recommended as a surgical option for HR due to high complication rate, however, positive outcomes of the Weil olsteotomy have been reported by Malerba et al.

Joint destructive procedures (MPT arthrodesis, arthroplasty, resection arthroplasty, interpositional arthroplasty) are indicated for end-stage HR. The Keller arthroplasty, first described in the early 20th century, suggested resection of the base of the proximal phalanx to decompress first MTPJ and improve dorsiflexion. With positive results reported in the series, complications associated with the Keller procedure include flail hallux, progressive hallux deformity and secondary metatarsalgia [31]. A prospective study of the Keller procedure carried out on 75 feet was performed by Love et al. with the average follow-up of 31 months. The operation was effective in improving joint pain in $91 \%$ with the overall patient satisfaction rate of $77 \%$. Cock-up deformity developed in $41 \%$ of the cases [32].

Interpositional arthroplasty involves insertion of a biologial spacer into the resected joint to improve range of motion and releive pain. Complications from this procedure are common, they include secondary metatarsalgia and first toe weakness [24]. No statistically significant differences were detected between resection and interpositional arthroplasties of first MTPJ by Schenk and colleagues in treatment of patients with Hattrup and Johnson grades II and III HR [33]. The arthroplasty technique described by Coughlin and Shurnas involved placement of a m. gracilis tendon bundle as a biologic spacer giving excellent pain relief and reliable function of the hallux. However, secondary metatarsalgia was revealed in $57 \%$ of the cases [6]. High complication rates pose a concern for the common use of resection and interpositional arthroplasties and also together with the development and refinement of new surgical techniques for HR. Although patient satisfaction rates are quoted as between $64 \%$ and $83 \%$ from MTPJ arthroplasty, failures would be difficult to address either with revision or arthrodesis due to inevitable bone loss. First- and second-generation silicone based implants were associated with high rates of component loosening, osteolysis and immune reactions to such implants [34, 35]. Subsequent implants which include metallic implants similar to hip and knee replacements have been plagued similarly with high loosening rates and high osteolysis rates [36, 37]. Pulavarti and colleagues reviewed functional results of 32 patients with implants arthroplasty at a mean followup of 3.9 years and noted implant instability in $33 \%$ of the cases with a revision required in $5.5 \%$ [36].

Gibson and Thomson reviewed results of first MTPJ arthroplasty and recorded bone resorption at the implant site in $49 \%$ within the first year postsurgery, and a revision procedure was produced in $15 \%$ of the cases within the first two years of surgery [37]. Nagy and colleagues reported poor midterm results of second-generation ceramic implants with survival of $68 \%$ [38]. Dawson-Bowling et al. presented a 8-year followup of 32 procedures that showed radiological evidence of component loosening in $52 \%$ of the cases with the reoperation rate of $26 \%$ [39].

Hemiarthroplasty of the proximal or the distal portion of first MTPJ with a metallic resurfacing component has also been desrcibed in literature. Promising results of a metallic resurfacing hemiarthroplasty of the proximal phalanx of 279 arthritic hallux MTPJ were presented by Townley and colleagues. Follow-up at 8 months to 33 years after surgery revealed good or excellent clinical results in $95 \%$ [40]. However, no one could reproduce the excellent outcomes with high rate of poor outcomes reported in literature. Konkel et al. observed implant instability of P1 articular surface in $100 \%$ of the patients at a followup of 37 to 105 months [41]. Raikin et al. compared the long-term clinical and radiographic outcomes of 21 metallic hemiarthroplasties and 27 arthrodeses of first MTPJ. The AOFAS and visual analogue pain scores and satisfaction appeared to be worse in the hemiarthroplasty group at a mean of 6.6 years with a revision surgery required in $24 \%$ of the hemiarthroplasties [42]. 
A synthetic cartilage implant device was developed to replace M1 articular surface. A multicenter prospective, randomized study compared the efficacy of this implant to a great toe arthrodesis for advancedstage HR in 202 patients enrolled at a mean followup of 2 years. VAS pain scores and the FAAM sports and activity of daily living subscores improved significantly in both groups. Reoperation was required in $9.2 \%$ of implant patients with dorsiflexion in first MTPJ significantly increased by $4^{\circ}$ on average [43]. Advantages of a synthetic cartilage implant include smaller incisions of M1 head to allow arthrodesis without significant loss of M1 length if needed. The promising results require further validation studies and long-term researches to assess implant survival.

Arthrodesis of the first MTPJ has been used since the mid-20th century. In 1952 McKeeve described unintentional ankylos is of the first MTPJ in one of his patients following infection. Surprisingly, the ankylosed joint was functioning with better result despite absence of motion at the affected joint. Premeditated ankylosis of the first MTPJ joint was described earlier by Wyeth (1887) and later by Clutton (1894) who contended that ankylosis in an ideal position would produce a permanent and very satisfactory result [44].

Fusion of the first MTPJ remains the gold standard treatment for advanced HR and multiple techniques have been described for MTPJ arthrodesis [11,
42, 45, 46 ]. In biomechanical studies, a lag screw and plate have been found to offer the most stable construct, The optimal position for first MTPJ fusion should be neutral rotation, $5^{\circ}-15^{\circ}$ of valgus and $10^{\circ}-15^{\circ}$ of dorsiflexion relative to the floor (or $20^{\circ}-$ $25^{\circ}$ relative to M1) [47, 48]. Goucher and Coughlin reported on 50 patients prospectively after first MTP arthrodesis using conical reamers and fixation with a lag screw and dorsal plate. Patients had a $96 \%$ satisfaction rate, $92 \%$ union rate and $4 \%$ revision rate at average 1.3-year follow-up [49]. Doty et al. reported $89 \%$ of good to excellent results in 49 patients who underwent a first MTPJ arthrodesis with bone consolidation achieved in $98 \%$ of the cases during an enrollment period of 1 year [50]. Bennett and Sabetta prospectively evaluated 200 patients who underwent first MTPJ arthrodeses utilizing a plate. Solid fusion occurred in $99 \%$ of the cases at a mean of 1-year follow-up with reoperation rate of $1 \%$ [51]. Brodsky et al. examined sports participation in 53 patients following first MTPJ fusion and found that patients were able to return to hiking $92 \%$ of the time, golf $80 \%$ of the time, tennis $75 \%$ of the time and jogging $75 \%$ of the time [52]. Although a jointdestructive procedure, first MTPJ arthrodesis is a successful surgical procedure that provides relief of pain, mechanical stability, functional improvements and allows individuals to return to most of their recreational sporting acitivities.

\section{CONCLUSION}

Hallux rigidus is a common degenerative joint condition of the foot that can cause considerable impairment of physical activities due to progressive stiffness at first MTPJ and local or wide-spread pain in the distal parts of the limb. Review of foreign literature revealed a great number of publications reporting nonoperative and operative treatment of the condition. The efficacy of non-operative modalities for HR was assessed by some authors as low at a longer term due to progression of the disease. Numerous grading systems used to describe the progressive level of joint arthritis suggest the lack of a unified approach and an accompanying treatment algorithm for HR. A joint sparing approach is the preferred option for both the patient and the surgeon and can be secured for early stages of HR with the efficacy decreasing and degeneration grade increasing. Isolated cheilectomy is normally offered for grades I and II HR and being used in conjunction with osteotomy of the proximal phalanx can be employed for grade III HR providing good functional outcomes and high patient satisfaction rate.
Cheilectomy can be also used in conjucntion with M1 osteotomy but the procedure is associated with a high rate of complications with secondary metatarsalgia being most common at a long-term follow-up. Maintaining an adequate length of M1 is important for preventing metatarsalgia and shortening osteotomy can be reserved for the cases with M1 longer that M2. First MTPJ arthrodesis remains the surgical treatment of choice for expressed arthritic joint providing reliable function recovery at a long term despite a joint destructive aspect of the procedure. There are discussions among medical community regarding benefits from first MTPJ arthrodesis providing predictable positive outcomes at the expense of movement in first MTPJ and normalizing gait biomechanics, and first MTPJ arthroplasty aimed at preservation of the parameters. Perspective surgical tendencies include the development and introduction of new implants to replace degenerative tissues of the first MTPJ. However, the innovations require scrupulous and long-term study to avoid adverse events like osteolysis and component loosening. There is also a greater 
risk of losing the bone stock making reoperations more challenging. A variety of surgical approaches are available for HR and the preferred treatment options would involve the use of isolated chelectomy or chelectomy in conjunction with P1 and/or M1 osteotomy and first MTPJ arthrodesis to ensure reliable functional outcomes for decades of practice with highlevel evidence support.

\section{REFERENCES}

1. Gould N., Schneider W., Ashikaga T. Epidemiological survey of foot problems in the continental United States. Foot Ankle, 1980, vol. 1, no. 1, pp. 810. DOI: 10.1177/107110078000100104.

2. Razik A., Sott A.H. Cheilectomy for Hallux Rigidus. Foot Ankle Clin., 2016, vol. 21, no. 3, pp. 451457. DOI: 10.1016/j. fcl.2016.04.006.

3. Roddy E., Thomas M.J., Marshall M., Rathod T., Myers H., Menz H.B., Thomas E., Peat G. The population prevalence of symptomatic radiographic foot osteoarthritis in community-dwelling older adults: cross-sectional findings from the clinical assessment study of the foot. Ann. Rheum. Dis., 2015, vol. 74, no. 1, pp. 156-163. DOI: 10.1136/annrheumdis-2013-203804.

4. Therman H., Becher C., Kilger R. Hallux rigidus treatment with cheilecomy, extensive plantar release, and additional microfracture technique. Techn. Foot Ankle Surg., 2004, vol. 3, pp. 210-217.

5. Root M., Orien W., Weed J. Normal and Abnormal Function of the Foot. Clinical Biomechanics. Los Angeles, Clinical Biomechanics Corp. 1977, vol. 2, 478 p.

6. Coughlin M.J., Shurnas P.J. Soft-tissue arthroplasty for hallux rigidus. Foot Ankle Int., 2003, vol. 24, no. 9, pp. 661-672. DOI: 10. 1177/107110070302400902.

7. Coughlin M.J., Shurnas P.S. Hallux rigidus: demographics, etiology, and radiographic assessment. Foot Ankle Int., 2003, vol. 24, no. 10, pp. 731-743. DOI: 10.1177/107110070302401002.

8. DuVries H. Static deformities. In: DuVries H., editor. Surgery of the Foot. St Louis (MO), Mosby, 1959, pp. 392-398.

9. Schnirring-Judge M., Hehemann D. The cheilectomy and its modifications. Clin. Podiatr. Med. Surg., 2011, vol. 28, no. 2, pp. 305327, viii. DOI: 10.1016/j.cpm.2011.03.004.

10.Feltham G.T., Hanks S.E., Marcus R.E. Age-based outcomes of cheilectomy for the treatment of hallux rigidus. Foot Ankle Int., 2001, vol. 22, no. 3, pp. 192-197. DOI: 10.1177/107110070102200304.

11.Coughlin M.J., Shurnas P.S. Hallux rigidus. Grading and long-term results of operative treatment. J. Bone Joint Surg. Am., 2003, vol. 85, no. 11, pp. 2072-2088.

12.Coughlin M.J., Shurnas P.S. Hallux rigidus. J. Bone Joint Surg. Am., 2004, vol. 86-A, no. Suppl. 1 (Pt. 2), pp. $119-130$. DOI: 10.2106/00004623-200409001-00003.

13.Roukis T.S., Landsman A.S., Ringstrom J.B., Kirschner P., Wuenschel M. Distally based capsule-periosteum interpositional arthroplasty for hallux rigidus. Indications, operative technique, and short-term follow-up. J. Am. Podiatr. Med. Assoc., 2003, vol. 93, no. 5, pp. 349-366. DOI: 10.7547/87507315-93-5-349.

14.Easley M.E., Davis W.H., Anderson R.B. Intermediate to long-term follow-up of medial-approach dorsal cheilectomy for hallux rigidus. Foot Ankle Int., 1999, vol. 20, no. 3, pp. 147-152. DOI: 10.1177/107110079902000302.

15.Lin J., Murphy G.A. Treatment of hallux rigidus with cheilectomy using a dorsolateral approach. Foot Ankle Int., 2009, vol. 30, no. 2, pp. 115-119. DOI: 10.3113/FAI-2009-0115.

16.Mann R.A., Coughlin M.J., DuVries H.L. Hallux rigidus: a review of the literature and a method of treatment. Clin. Orthop. Relat. Res., 1979, no. 142, pp. 57-63.

17.Roukis T.S. The need for surgical revision after isolated cheilectomy for hallux rigidus: a systematic review. J. Foot Ankle Surg., 2010, vol. 49, no. 5, pp. 465-470. DOI: 10.1053/j.jfas.2010.06.013.

18.Mulier T., Steenwerckx A., Thienpont E., Sioen W., Hoore K.D., Peeraer L., Dereymaeker G. Results after cheilectomy in athletes with hallux rigidus. Foot Ankle Int., 1999, vol. 20, no. 4, pp. 232-237. DOI: 10.1177/107110079902000405.

19.Nawoczenski D.A., Ketz J., Baumhauer J.F. Dynamic kinematic and plantar pressure changes following cheilectomy for hallux rigidus: a mid-term follow-up. Foot Ankle Int., 2008, vol. 29, no. 3, pp. 265-272. DOI: 10.3113/FAI.2008.0265.

20.Seibert N.R., Kadakia A.R. Surgical management of hallux rigidus: cheilectomy and osteotomy (phalanx and metatarsal). Foot Ankle Clin., 2009, vol. 14, no. 1, pp. 9-22. DOI: 10.1016/j.fcl.2008.11.002.

21.Kim P.H., Chen X., Hillstrom H., Ellis S.J., Baxter J.R., Deland J.T. Moberg osteotomy shifts contact pressure plantarly in the first metatarsophalangeal joint in a biomechanical model.Foot AnkleInt., 2016, vol.37, no.1, pp. 96-101.DOI:10.1177/1071100715603513.

22.Thomas P.J., Smith R.W. Proximal phalanx osteotomy for the surgical treatment of hallux rigidus. Foot Ankle Int., 1999, vol. 20, no. 1, pp. 3-12. DOI: 10.1177/107110079902000102.

23.O'Malley M.J., Basran H.S., Gu Y., Sayres S., Deland J.T. Treatment of advanced stages of hallux rigidus with cheilectomy and phalangeal osteotomy. J. Bone Joint Surg. Am., 2013, vol. 95, no. 7, pp. 606-610. DOI: 10.2106/JBJS.K.00904.

24.Lau J.T., Daniels T.R. Outcomes following cheilectomy and interpositional arthroplasty in hallux rigidus. Foot Ankle Int., 2001, vol. 22, no. 6, pp. 462-470. DOI: 10.1177/107110070102200602.

25.Waizy H., Czardybon M.A., Stukenborg-Colsman C., Wingenfeld C., Wellmann M., Windhagen H., Frank D. Mid- and longterm results of the joint preserving therapy of hallux rigidus. Arch. Orthop. Trauma Surg., 2010, vol. 130, no. 2, pp. 165-170. DOI: 10.1007/s00402-009-0857-1.

26.Dickerson J.B., Green R., Green D.R. Long-term follow-up of the Green-Watermann osteotomy for hallux limitus. J. Am. Podiatr. Med. Assoc., 2002, vol. 92, no. 10, pp. 543-554. DOI: 10.7547/87507315-92-10-543.

27.Bryant A.R., Tinley P., Cole J.H. Plantar pressure and joint motion after the Youngswick procedure for hallux limitus. J. Am. Podiatr. Med. Assoc., 2004, vol. 94, no. 1, pp. 22-30. DOI: 10.7547/87507315-94-1-22.

28.Derner R., Goss K., Postowski H.N., Parsley N. A plantar-flexor-shortening osteotomy for hallux rigidus: a retrospective analysis. J. Foot Ankle Surg., 2005, vol. 44, no. 5, pp. 377-389. DOI: 10.1053/j.jfas.2005.07.010. 
29.Malerba F., Milani R., Sartorelli E., Haddo O. Distal oblique first metatarsal osteotomy in grade 3 hallux rigidus: a long-term followup. Foot Ankle Int., 2008, vol. 29, no. 7, pp. 677-682. DOI: 10.3113/FAI.2008.0677.

30.Roukis T.S. Clinical outcomes after isolated periarticular osteotomies of the first metatarsal for hallux rigidus: a systematic review. J. Foot Ankle Surg., 2010, vol. 49, no. 6, pp. 553-560. DOI: 10.1053/j.jfas.2010.08.014.

31.Beertema W., Draijer W.F., Van Os J.J., Pilot P. A retrospective analysis of surgical treatment in patients with symptomatic hallux rigidus: long-term follow-up. J. Foot Ankle Surg., 2006, vol. 45, no. 4, pp. 244-251. DOI: 10.1053/j.jfas.2006.04.006.

32.Love T.R., Whynot A.S., Farine I., Lavoie M., Hunt L., Gross A. Keller arthroplasty: a prospective review. Foot Ankle, 1987, vol. 8, no. 1, pp. 46-54. DOI: 10.1177/107110078700800110.

33.Schenk S., Meizer R., Kramer R., Aigner N., Landsiedl F., Steinboeck G. Resection arthroplasty with and without capsular interposition for treatment of severe hallux rigidus. Int. Orthop., 2009, vol. 33, no. 1, pp. 145-150. DOI: 10.1007/s00264-007-0457-z.

34.Cracchiolo A. 3rd, Weltmer J.B. Jr., Lian G., Dalseth T., Dorey F. Arthroplasty of the first metatarsophalangeal joint with a doublestem silicone implant. Results in patients who have degenerative joint disease failure of previous operations, or rheumatoid arthritis. J. Bone Joint Surg. Am., 1992, vol. 74, no. 4, pp. 552-563.

35.Sebold E.J., Cracchiolo A. 3rd. Use of titanium grommets in silicone implant arthroplasty of the hallux metatarsophalangeal joint. Foot Ankle Int., 1996, vol. 17, no. 3, pp. 145-151. DOI: 10.1177/107110079601700305.

36.Pulavarti R.S., McVie J.L., Tulloch C.J. First metatarsophalangeal joint replacement using the bio-action great toe implant: intermediate results. Foot Ankle Int., 2005, vol. 26, no. 12, pp. 1033-1037. DOI: 10.1177/107110070502601206.

37.Gibson J.N., Thomson C.E. Arthrodesis or total replacement arthroplasty for hallux rigidus: a randomized controlled trial. Foot Ankle Int., 2005, vol. 26, no. 9, pp. 680-690. DOI: 10.1177/107110070502600904.

38.Nagy M.T., Walker C.R., Sirikonda S.P. Second-generation ceramic first metatarsophalangeal joint replacement for hallux rigidus. Foot Ankle Int., 2014, vol. 35, no. 7, pp. 690-698. DOI: 10.1177/1071100714536539.

39.Dawson-Bowling S., Adimonye A., Cohen A., Cottam H., Ritchie J., Fordyce M. MOJE ceramic metatarsophalangeal arthroplasty: disappointing clinical results at two to eight years. Foot Ankle Int., 2012, vol. 33, no. 7, pp. 560-564. DOI: 10.3113/FAI.2012.0560.

40.Townley C.O., Taranow W.S. A metallic hemiarthroplasty resurfacing prosthesis for the hallux metatarsophalangeal joint. Foot Ankle Int., 1994, vol. 15, no. 11, pp. 575-580. DOI: 10.1177/107110079401501101.

41.Konkel K.F., Menger A.G. Mid-term results of titanium hemi-great toe implants. Foot Ankle Int., 2006, vol. 27, no. 11, pp. $922-929$. DOI: $10.1177 / 107110070602701110$.

42.Raikin S.M., Ahmad J., Pour A.E., Abidi N. Comparison of arthrodesis and metallic hemiarthroplasty of the hallux metatarsophalangeal joint. J. Bone Joint Surg. Am., 2007, vol. 89, no. 9, pp. 1979-1985.

43.Baumhauer J.F., Singh D., Glazebrook M., Blundell C., De Vries G., Le I.L.D., Nielsen D., Pedersen M.E., Sakellariou A., Solan M., Wansbrough G., Younger A.S.E., Daniels T.; for and on behalf of the CARTIVA Motion Study Group. Prospective, randomized, multi-centered clinical trial assessing safety and efficacy of a synthetic cartilage implant versus first metatarsophalangeal arthrodesis in advanced hallux rigidus. Foot Ankle Int., 2016, vol. 37, no. 5, pp. 457-469. DOI: 10.1177/1071100716635560.

44. Kelikian H. Arthrodesing operations. In: Kelikian H. Hallux valgus, allied deformities of the forefoot and metatarsalgia. Philadelphia and London, W.B. Saunders Company. 1965, pp. 236-248. DOI: 10.1002/bjs.1800530141.

45.Kumar S., Pradhan R., Rosenfeld P.F. First metatarsophalangeal arthrodesis using a dorsal plate and a compression screw. Foot Ankle Int., 2010, vol. 31, no. 9, pp. 797-801. DOI: 10.3113/FAI.2010.0797.

46.Ho B., Baumhauer J. Hallux rigidus. EFORT Open Rev., 2017, vol. 2, no. 1, pp. 13-20. DOI: 10.1302/2058-5241.2.160031.

47.Politi J., John H., Njus G., Bennett G.L., Kay D.B. First metatarsal-phalangeal joint arthrodesis: a biomechanical assessment of stability. Foot Ankle Int., 2003, vol. 24, no. 4, pp. 332-337. DOI: 10.1177/107110070302400405.

48.Hyer C.F., Glover J.P., Berlet G.C., Lee T.H. Cost comparison of crossed screws versus dorsal plate construct for first metatarsophalangeal joint arthrodesis. J. Foot Ankle Surg., 2008, vol. 47, no. 1, pp. 13-18. DOI: 10.1053/j.jfas.2007.08.016.

49.Goucher N.R., Coughlin M.J. Hallux metatarsophalangeal joint arthrodesis using dome-shaped reamers and dorsal plate fixation: a prospective study. Foot Ankle Int., 2006, vol. 27, no. 11, pp. 869-876. DOI: 10.1177/107110070602701101.

50.Doty J., Coughlin M., Hirose C., Kemp T. Hallux metatarsophalangeal joint arthrodesis with a hybrid locking plate and a plantar neutralization screw: a prospective study. Foot Ankle Int., 2013, vol. 34, no. 11, pp. 1535-1540. DOI: 10.1177/1071100713494779.

51.Bennett G.L., Sabetta J. First metatarsalphalangeal joint arthrodesis: evaluation of plate and screw fixation. Foot Ankle Int., 2009, vol. 30, no. 8, pp. 752-757. DOI: 10.3113/FAI.2009.0752.

52.Brodsky J.W., Passmore R.N., Pollo F.E., Shabat S. Functional outcome of arthrodesis of the first metatarsophalangeal joint using parallel screw fixation. Foot Ankle Int., 2005, vol. 26, no. 2, pp. 140-146. DOI: 10.1177/107110070502600205.

Received: 10.12.2018

\section{Information about the authors:}

1. Denis V. Ilchenko,

European Clinic of Sports Traumatology and Orthopaedics, Moscow, Russian Federation,

Email: dilchenko@emcmos.ru

2. Andrey A. Kardanov, M.D., Ph.D., Professor,

Peoples' Friendship University of Russia, Moscow, Russian Federation,

European Clinic of Sports Traumatology and Orthopaedics, Moscow, Russian Federation,

Email: akardanov@emcmos.ru

3. Andrey V. Korolev, M.D., Ph.D., Professor,

European Clinic of Sports Traumatology and Orthopaedics (ECSTO), Moscow, Russian Federation,

Peoples’ Friendship University of Russia, Moscow, Russian Federation,

Email: akorolev@emcmos.ru 